\title{
Abstracts from the ASENT 2006 Annual Meeting March 8-11, 2006
}

\author{
Duloxetine in the Management of Diabetic \\ Peripheral Neuropathic Pain: Response Profile \\ Y Pritchett, B McCarberg, J Watkin, A Chappell, \\ M Robinson \\ Eli Lilly and Company and University of California- \\ San Diego
}

Introduction: Analyses examine the response rate at endpoint, as well as time course of response, in patients receiving duloxetine for management of diabetic peripheral neuropathic pain (DPNP).

Methods: Data were pooled from 3 double-blind, randomized, placebo-controlled 12-week trials in patients with DPNP $\geq 6$ months duration, and without depression. Study $1(N=$ 457) compared duloxetine $20 \mathrm{mg}$ once daily (QD), $60 \mathrm{mg}$ QD, $60 \mathrm{mg}$ twice daily (BID), and placebo; Studies $2(N=334)$ and $3(N=348)$ compared duloxetine $60 \mathrm{mg}$ QD and $60 \mathrm{mg}$ BID with placebo. Ethics committees approved the study protocol in accordance with Declaration of Helsinki principles. Patients provided written informed consent prior to study participation. Treatment response was defined a priori as $30 \%$ reduction in the primary efficacy measure, 24-hour average pain severity. Analysis was replicated using alternative criteria (50\% reduction or 2-point reduction).

Results: Endpoint response rates were significantly higher among patients receiving duloxetine (60 mg QD or $60 \mathrm{mg}$ BID) than those receiving placebo, regardless of chosen response criterion. The proportion of patients responding (30\% reduction in 24-hour average pain severity) to duloxetine was statistically greater than to placebo Week 1 and all subsequent visits. Similar results were obtained for the visitwise sustained response rate. Within the group with a sustained response at Week 12, the proportion of patients first exhibiting a response at Weeks 1 or 2 was higher in the duloxetine groups $(60 \mathrm{mg} \mathrm{QD}, 65.0 \%$; $60 \mathrm{mg}$ BID, $62.0 \%$ ) when compared with the placebo group $(40.2 \%)$.

Conclusion: Patients with DPNP receiving duloxetine 60 mg QD or $60 \mathrm{mg}$ BID had significantly higher treatment response rates compared with patients receiving placebo, regardless of response criterion. Response to duloxetine treatment tended to occur early, with approximately $30 \%$ of patients responding at Week 1. Among duloxetine-treated patients with a sustained treatment response at Week 12, over $60 \%$ had maintained responder status for 10 to 11 weeks.

\section{Evaluation of Duloxetine Effectiveness in the Management of DPNP with Respect to Baseline Characteristics}

\section{Y Pritchett, J Xu, B Rotz, A Chappell, J Wernicke Eli Lilly and Company}

Objective: To examine impact of age, gender, origin, duration of diabetic neuropathy, type of diabetes, and pain severity on treatment outcome of duloxetine (DLX) in the management of diabetic peripheral neuropathic pain (DPNP).

Methods: Data from 3 12-week, multicenter, double-blind, placebo-controlled studies (1-3) were pooled. In Study 1, 457 patients with DPNP were randomly assigned to DLX $20 \mathrm{mg}$ QD, $60 \mathrm{mg}$ QD, $60 \mathrm{mg}$ BID, or placebo. In Studies 2 and 3, 334 and 348 patients, respectively, were randomly assigned to DLX $60 \mathrm{mg}$ QD, $60 \mathrm{mg} \mathrm{BID}$, or placebo. DLX $20 \mathrm{mg}$ QD was not included in the analyses due to its ineffectiveness found in Study 1 . Primary efficacy measure was the weekly mean score of 24-Hour Average Pain Severity collected by patient's diary on 11-point Likert scale. Subgroup impact was evaluated for combined DLX doses with references to placebo group.

Results: DLX $60 \mathrm{mg}$ QD and $60 \mathrm{mg}$ BID significantly improved weekly mean scores of 24-Hour Average Pain Severity in each study $(p<0.001)$. There were no statistical significant $(p<0.1)$ interactions of treatment with age $(<65$ or $\geq 65$ ), gender (male or female), origin (Caucasian or other), duration of diabetic neuropathy $(<2,2-<6$, or $\geq 6$ years $)$, type of diabetes (Type I or Type II) with an exception for pain severity (BPI average pain $<6$ or $\geq 6$ ). DLX effect was observed for both severe and less severe groups with more separation on the severe group. The superiority of DLX over placebo was observed within almost each of the stratum with the exception of Type I patient group, which demonstrated the advantage of DLX treatment with $p=0.123$. The magnitude of change was slightly higher in Type II patients than in those with Type I; however, Type I group sample size was much smaller which may account for the statistical insignificance. DLX was safe and well tolerated with less than $20 \%$ discontinuation due to AEs, while showing no interference with diabetic control.

Conclusion: These studies suggested that DLX was effective and safe in the treatment of DPNP, and its effectiveness was invariant with respect to baseline conditions.

Funding provided by Eli Lilly and Company.

\section{Cardiovascular Effects of Duloxetine: Preclinical and Clinical Findings}

M Detke, S Iyengar, J Henck, F Bymaster, J Callaghan, M Knadler, A Chappell, J Wernicke, M Thase

Eli Lilly and Company, Indiana University School of Medicine, and University of Pittsburgh Medical Center

Background: We summarize cardiac effects of duloxetine based on in vitro and animal studies, and human trials including higher doses and chronic treatment.

Methods: The affinity of duloxetine for cardiac ion channels was determined in vitro in a stably expressed human cell line. Cardiovascular parameters were evaluated in single- and repeat-dose studies in rats and dogs. In humans, cardiovascular safety was analyzed in (1) healthy volunteers receiving duloxetine up to $400 \mathrm{mg} / \mathrm{d}$; (2) 8 placebo-controlled MDD trials of duloxetine $(40-120 \mathrm{mg} / \mathrm{d})$ for 8-9 weeks, compared with paroxetine or fluoxetine in 6 trials; (3) 52-week, open-label duloxetine study (80-120 mg/d); (4) 12-week MDD study of duloxetine versus venlafaxine.

Results: Duloxetine, at the maximum unbound plasma concentration observed clinically, had no adverse effect on the human cardiac ion channels tested in vitro. Blood pressure (BP) and heart rate (HR) were not significantly altered in conscious 
animals following single oral doses of 7 or $20 \mathrm{mg} / \mathrm{kg}$ in rats and $10 \mathrm{mg} / \mathrm{kg}$ in dogs. Cardiac rhythm, conduction, and HR were unaffected in dogs by up to 1 year of treatment with 3,10 , or $30 \mathrm{mg} / \mathrm{kg}$. In healthy volunteers, duloxetine had a modest effect on BP and HR elevation with no prolongation of QTc interval. In patients with MDD, small increases in HR and BP were observed. Although there were statistically significant differences in HR from baseline to endpoint between duloxetine versus fluoxetine or paroxetine, changes were clinically insignificant. In the study of duloxetine $(60-120 \mathrm{mg} /$ day) and venlafaxine (75-225 mg/day) in MDD, no statistically significant differences occurred in elevated HR and BP except for significantly more venlafaxine patients with sustained systolic BP during the first 6 weeks.

Discussion: Consistent with the lack of effect on human cardiac ion channel binding in vitro, and the lack of effect on conduction parameters in repeat-dose dog studies, no QTc prolongation was seen in humans. Consistent with the HR and BP findings in animals, humans had a few small effects with these measures. In summary, duloxetine has a good cardiovascular safety profile, possibly due to its lack of affinity for crucial cardiac ion channels and/or other unknown factors.

Funding provided by Eli Lilly and Company.

\section{Neurosurgical Treatment of Intractable Tourette's Syndrome: A Scientific Clinical Review of Literatures}

\section{Meratee, J Chung, J Schweitzer}

\section{Kaiser Permanente Southern California Medical Center}

Background: Tourette's syndrome (TS) is an inherited neuropsychiatric disorder characterized by fluctuating motor and vocal tics that starts in childhood. Diagnostic criteria for TS include the presence of multiple motor tics and one or more vocal tics, both of which must exceed a year's duration. Pharmacotherapy has been the mainstay of the treatment. However, some TS patients will not respond to conventional medications. They might develop residual debilitating symptoms. The current article examines the reported experience with neurosurgical treatment of intractable Tourette's syndrome.

Design/Method: All articles and textbooks containing descriptions of Tourette's syndrome and its surgical treatment were reviewed. All related articles in PubMed were searched using these words: Tourette's syndrome, TS, Tourette, Gilles de la Tourette, tic, tics, surgical treatment of tic disorders, surgical intervention of Tourrete's syndrome, ablative surgery in Tourette's syndrome, stereotactic surgery of Tourette's syndrome. Only articles published in English were reviewed. In total, 11 articles and 2 textbooks were found describing the experimental surgery of refractory Tourette's syndrome. All data about patient's history, surgical treatment, outcome and side effects were collected and reviewed.

Results: Intractable Tourette's syndrome may represent a special subgroup of the tic range. A variety of experimental procedures have been performed in an attempt to treat intractable Tourette's syndrome, including: 1) frontal lobe operation (i.e., frontal lobotomy and bimedial frontal leucotomy), 2) limbic system operation (i.e., anterior cingulotomy, limbic leucotomy), 3) a novel multisite operation (i.e., anterior cingulotomy combined with infrathalamic lesions), 4) thalamic operation (i.e., bilateral coagulation of rostral infrathalamic and medial thalamic nuclei), and 5) a cerebellar operation (i.e., bilateral cerebellar dentatomy). There are serious uncertainties regarding any experimental neurosurgical procedures. However, in the case of severe, intractable Tourette's syndrome, these appropriate concerns must be weighed against the risks of conventional therapies, including tardive dyskinesia. Patients who suffer from severe Tourette's syndrome, and are debilitated by their symptoms, and have failed an exhaustive range of conventional pharmacologic therapies could be candidates for an experimental treatment.

Conclusion: Case reports in the literature provide only anecdotal evidence supporting the efficacy and safety of neurosurgical treatment of Tourette's syndrome. There is no convincing evidence showing that any particular neurosurgical procedure is best for Tourette's syndrome. If experimental neurosurgery for Tourette's syndrome is to continue, then guidelines should be developed regarding patient and operation selection. Also, accurate clinical measurement should be applied preoperatively and postoperatively to monitor long-term outcome.

\section{The Role of Microglia and NADPH Oxidase in Traumatic Spinal Cord Injury \\ K Byrnes, B Stoica, S Di Giovanni, A De Biase, S Knoblach, E Hoffman, A Faden \\ Georgetown University and Children's National Medical Center}

Spinal cord injury (SCI) results in delayed biochemical changes that contribute to secondary tissue damage and dysfunction. The microglial-induced inflammatory response, long implicated in secondary tissue damage after SCI, includes release of various factors known to be toxic to neurons. However, the response of microglia and their subsequent role remains controversial and poorly understood. The present study evaluated the acute and chronic gene expression profiles in rats after traumatic SCI. Using anchor genes selected from the literature because of their relationship to microglial-induced inflammation, high density microarray expression profiles were examined after contusion. Two temporally correlated clusters were identified: one was expressed immediately after injury, peaking between 4 and 24 hours and declining to baseline or below baseline levels; the second was expressed chronically after injury, and remained elevated up to 6 months after injury. Expression profiles for genes of each group were confirmed using realtime quantitative PCR, immunoblotting, and immunohistochemistry. One of the genes chronically expressed from 24 hours to 6 months postinjury was $\mathrm{p} 22^{\text {phox }}$, a component of the NADPH oxidase enzyme. To investigate the role of this chronically expressed gene after SCI and the effect of modification of its activity, the NADPH oxidase inhibitor apocynin was added to purified microglia cultures and microglial activation after LPS stimulation was investigated. Inhibition of $\mathrm{p} 22^{\text {phox }} / \mathrm{NADPH}$ oxidase resulted in significant suppression of microglial proliferation, nitric oxide production, and microglial-induced neurotoxicity. This study demonstrates the potential utility of microarray technology in identifying possible targets for future therapeutics. Additionally, it identifies p22 $22^{\text {phox }}$ and NADPH as potential components of microglial-induced neurotoxicity, with implications for neuroprotective therapy.

\section{Noninvasive Measurement and Quantitation of Brain Iron in Parkinson's Disease Using Adiabatic T2 $\rho$ and T1 $\rho$ High Field MRI Techniques}

S Michaeli, D Sorce, G Öz, K Ugurbil, M Garwood, P Tuite University of Minnesota

Postmortem demonstration of increased iron in the substantia nigra $(\mathrm{SN})$ is a well- appreciated finding in Parkin- 
son's disease (PD). It is thought that this iron may facilitate the generation of free radicals which are thought to play a role in dopamine neuronal loss. To date, however, researchers have been unable to confirm an in vivo difference of iron between those with $\mathrm{PD}$ and control subjects using magnetic resonance imaging (MRI). This may be due to the limitations of T1 and T2: two conventional MRI techniques that have been employed. Here, novel T2 $\rho$ and T1 $\rho$ MRI relaxation methods were used for the measurement of iron load and distribution $[1,2,3]$. T2 $\rho$ measurements are indicative of tissue iron content and distribution and measure molecular motion in local susceptibility gradients. $\mathrm{T} 1 \rho$ measurements, on the other hand, can be used to assess cellular loss. When applied at high magnetic fields these two methods may provide a noninvasive and reliable handle on iron accumulation and neuronal loss in PD. In our 4 Tesla magnet study, we found a significant change of the T $1 \rho$ and $\mathrm{T} 2 \rho$ relaxation time constants (both the distribution and absolute values) in the PD group versus controls. Relaxogram analysis of the T $2 \rho$ and T $1 \rho$ measurements demonstrated increased water and iron content, as well as changes in iron distribution in the SN. Therefore, high resolution MRI with T2 $\rho$ and $\mathrm{T} 1 \rho$ provide unique information in Parkinson's disease patients as compared to conventional T1 and T2 measurements. This information may prove useful in evaluating the pathogenesis and severity of PD. Future studies are underway to correlate T1 $\rho, \mathrm{T} 2 \rho$, and T2 findings with simultaneous spectroscopy measurements of absolute concentration of NAA [4] to further assess iron accumulation and neuronal loss in controls and those with PD.

References: [1] Michaeli S, Sorce DJ, Idiyatullin D, Ugurbil K, Garwood M. J Magn Reson 169:293-299 (2004); [2] Michaeli S, Grohn H, Grohn O, Sorce DJ, Kauppinen R, Springer C, Ugurbil K, Garwood M. Magn Reson Med 53:823829 (2005); [3] Michaeli S, Sorce DJ, Springer C, Ugurbil K, Garwood M. PISMRM, 2005; [4] Öz G, Terpstra M, Tkaĉ I, Aia P; Lowary J, Tuite P, Gruetter G, MRM, in press.

This work was supported by NIH grant RR08079, the Keck Foundation, and the Mind Institute.

\section{NIH Countermeasures Against Chemical Threats (CounterACT) Research Program}

D Jett

\section{National Institutes of Neurological Disorders and Stroke}

The NIH is taking a leadership role in pursuing the development of new and improved medical countermeasures designed to prevent, diagnose, and treat the conditions caused by potential and existing chemical agents of terrorism. In addition, many of the same chemicals posing a threat as terrorist agents may also be released from transportation and storage facilities by industrial accidents or during a natural disaster. The NIH has developed a comprehensive Countermeasures Against Chemical Threats (CounterACT) Research Network that includes Research Centers of Excellence, individual research projects, Small Business Grants and Contracts, and other programs (see http://www.ninds.nih.gov/ funding/research/counterterrorism/index.htm). The network will conduct basic, translational, and clinical research aimed at the discovery and/or identification of better therapeutic and diagnostic medical countermeasures against chemical threat agents. The overarching goal of this research program is to enhance our diagnostic and treatment response capabilities during an emergency. The civilian chemical threat spectrum now includes chemical warfare agents, toxic industrial chemicals, toxins and other chemicals. Many of the highest priority chemical threat agents target the nervous system and require new and improved neurotherapeutics that can be used as safe and rapid medical countermeasures during a mass casualty situation. Examples of these chemical agents include anticholinesterase nerve "gases" such as sarin or VX, metabolic poisons such as cyanide, and biological neurotoxins such as saxitoxin. The type of research supported within the CounterACT research program includes: (1) mechanistic research to identify targets for therapeutic/diagnostic development, (2) development of in vitro and animal models for efficacy screening of therapeutics and diagnostic tools, (3) efficacy screening of therapeutics/diagnostics using new and validated in vitro and animal models, (4) advanced efficacy studies with appropriate animal models including nonhuman primates, and (5) clinical studies, including trials, when appropriate. Special consideration will be given to research relevant to people who are particularly vulnerable, including the young, the elderly, and individuals with preexisting medical conditions.

Interested parties are encouraged to contact Dr. David A. Jett, via email at jettd@ninds.nih.gov or by telephone at (301) 496-6035 for more information and funding opportunities within the NIH CounterACT research program.

\section{Dose Decrease and Other Correlates of Relapse in Patients with Schizophrenia or Schizoaffective Disorder During Olanzapine Drug Therapy}

W Deberdt, J Csernansky, P Buckley, J Peiskens, I Lipkovich, S Kollack-Walter, J Houston, Y Zhang, H Liu-Siefert

Eli Lilly and Company, Washington University School of Medicine, Medical College of Georgia, The Catholic

University of Leuven, and Virginia Polytechnic Institute and State University

Purpose: To assess dose decrease and other variables as correlates of relapse during olanzapine treatment.

Methods: In two 28-week, randomized, double-blind clinical trials, a Cox proportional hazards model was used for post hoc analysis of potential correlates of relapse (defined as $\geq 20 \%$ worsening on PANSS total and CGI-Severity $\geq 3$ ) among patients $(N=271)$ who responded to 8 weeks of olanzapine treatment (10-20 mg/day). Variables examined included demographics, illness characteristics, baseline symptoms, symptom changes, dose, adverse events, and functioning.

Results: Patients with a lower last dose relative to that of the preceding visit interval were 4 times more likely to relapse during that visit interval than patients without a lower dose $(p<0.001)$. A similar finding was observed for a decrease in interval modal dose and risk for relapse, although this relationship was more predictive of relapse in the next visit interval $(p=0.027)$. A dose decrease in women was not associated with relapse $(p=0.922)$, whereas a dose decrease in men did predict relapse $(p<0.001)$ Relapse also correlated with the emergence or worsening of a psychiatric adverse event during the same visit interval as relapse $(p<0.001)$, and during the preceding visit interval $(p=0.007)$. The occurrence of a nonpsychiatric adverse event was not associated with relapse.

Conclusion: Dose decrease was a significant predictor of relapse in male patients. Psychiatric adverse events were also a significant predictor of relapse. Symptom worsening and dose decrease are both clinical measures that call for closer monitoring of patients due to increased likelihood of relapse.

Funded by Eli Lilly and Company. 
Prediction of Combined Symptomatic and Functional Outcome in Patients with Schizophrenia or Schizoaffective Disorder

I Lipkovich, ${ }^{*}$ W Deberdt,* PF Buckley, ${ }^{\dagger}$ JG Csernansky,

J Peuskens, ${ }^{\S}$ S Kollack-Walker,* JP Houston, ${ }^{*}$ M Rotelli*

*Lilly Research Laboratories, Eli Lilly and Company, Indianapolis IN, USA; ${ }^{\dagger}$ Department of Psychiatry, Medical College of Georgia, Augusta GA, USA; ${ }^{\ddagger}$ Department of Psychiatry, Washington University School of Medicine, St. Louis MO, USA; ${ }^{\S}$ The Catholic University of Leuven, University Centre St. Jozef, Kortenberg, Belgium

Purpose: An earlier analysis of data from six randomized, active-control studies involving 1449 patients identified five distinct clusters characterized by different combinations of psychiatric and functional outcomes. We explored baseline demographics, disease characteristics, early symptom response, treatment, and adverse events as possible predictors of clusters representing best and worst clinical outcomes.

Methods: At 6-month endpoint in combined treatment groups, good outcome (Cluster A) was associated with good functioning and limited psychopathology. Poor outcome was associated with poor functioning and moderate (Cluster D) or severe (Cluster E) psychopathology. Stepwise logistic regression was used to construct predictive models of cluster membership $(N=1260)$ for baseline predictors and with $2 / 4 / 8$ weeks of treatment. Odds ratios were adjusted for study effects.

Results: Cluster A baseline predictors included female gender and higher levels of occupational and psychosocial functioning. Greater improvement across PANSS factors during early treatment also predicted good outcome. Cluster $\mathrm{D}$ baseline predictors included earlier onset of illness, older age, pseudoparkinsonism, and worse occupational and psychosocial functioning; subsequent worsening in PANSS depression and positive factor scores and in functioning predicted poor outcome for Cluster D. Predictors of Cluster E included earlier onset of illness, non-olanzapine treatment, and higher scores on the PANSS depression, hostility, and positive factors; subsequent worsening in PANSS disorganization, negative, and positive factor scores was predictive of poor outcome for Cluster E.

Conclusion: Early symptom improvement/worsening was predictive of outcome. Early monitoring of psychiatric symptoms and functioning may lead to better therapeutic decisions based on individual characteristics.

Funded by Eli Lilly and Company.

\section{Antiepileptic Drugs Do Not Affect 5-HT1A Receptor Binding Measured by Positron Emission Topography}

W Theodore, G Giovacchini, A Bagic, P Herscovitch, $\mathrm{R}$ Carson

National Institutes of Health, Clinical Epilepsy Section

Objective: To study the effect of antiepileptic drugs on $5-\mathrm{HT}_{1 \mathrm{~A}}$ receptor binding in patients with temporal lobe epilepsy.

Background: 5- $\mathrm{HT}_{1 \mathrm{~A}}$ receptor binding is reduced in patients with temporal lobe epilepsy. Animal models show serotonergic effects of antipepileptic drugs including carbamazepine and lamotrigine.

Methods: We studied 31 patients and 10 normal controls. Patients with structural lesions, progressive neurological disorders, or taking other medications were excluded. None had a seizure for at least two days before PET. $\left[{ }^{18} \mathrm{~F}\right] \mathrm{FCWAY}$ PET was performed on a GE Advance scanner with continuous EEG monitoring. Functional images of the distribution volume $(V)$ were generated. Anatomic regions of interest were applied to coregistered PET images, after correction for partial volume effect.

Results: Patients had significantly higher $\left[{ }^{18} \mathrm{~F}\right] \mathrm{FCWAY}$ free fraction $\left(f_{1}\right)$ than controls. 5HT1A receptor binding was reduced in temporal lobe epileptic foci after partial volume correction. There were no AED effects on interictal $\left[{ }^{18} \mathrm{~F}\right] \mathrm{FCWAY}$ binding after correction for plasma free fraction. $\left[{ }^{18} \mathrm{~F}\right] \mathrm{FCWAY}$ $V / f_{1}$ reduction in epileptic foci was not affected by AEDs.

Conclusions: Antiepileptic drugs do not appear to have significant effects on 5HT1A receptor binding. Plasma free fractions should be measured when PET receptor studies are performed in patients with epilepsy.

\section{Measuring Cortical Acetylcholine Esterase Activity by PET in Dementia: Clinical Correlates \\ K Herholz, S Weisenbach, R Hilker, W Heiss \\ Max-Planck-Institute for Neurological Research, Cologne, Germany}

The acetylcholine analogue C-11- $N$-methyl-4-piperidinylacetate (MP4A) is a tracer for positron emission tomography (PET) to investigate the integrity of the cerebral cholinergic system. It is a substrate of acetylcholine esterase (AChE), which is associated with cholinergic axons in human cortex. We used this technique to determine whether cortical reductions of AChE activity in dementia are associated with clinical symptoms and progression.

We examined the following age-matched subjects: 12 normal controls, 8 mild cognitive impairment (MCI, MMSE $27 \pm 2$ ), 10 mild Alzheimer's disease (AD, MMSE $21 \pm 4$ ), 10 Parkinson's disease dementia (PDD, MMSE $21 \pm 6$ ), and 10 non-demented PD (MMSE $27 \pm 2$ ). Cortical AChE activity was reduced significantly in all demented subjects and in those four MCI patients, who progressed to dementia within 18 months. The most severe reduction (by $30 \pm 5 \%$ ) was seen in PDD, followed by AD (23 \pm $2 \%)$ and nondemented PD $(12 \pm 2 \%, p<0.01$ compared to controls). Thus, the manifestation of dementia in PD was associated with a significant reduction of cortical AChE activity, which was most severe in the left inferior parietal lobule, the left precental gyrus, and the right posterior cingulate gyrus (SPM, $p<$ 0.001 ). In mild to moderate $\mathrm{AD}$, regional $\mathrm{AChE}$ activity in temporal, parietal, and frontal association cortices was associated with attention-dependent test performance (Rey-Osterrieth figure copy, digit span forward and reverse, visual span reverse).

We conclude that a reduction of cortical AChE activity is a common feature of Alzheimer's and Parkinson's dementia and appears to be related mainly to impairment of attention. It is present very early during the course of the disease, even at a predementia stage. It is likely to indicate functional impairment of the synapses or of axonal transport of ascending cholinergic neurons.

\section{Development of 1-Octanol as a Novel Therapy for the Treatment of Ethanol-Responsive Essential Tremor}

F Nahab, M Hallett

$$
\begin{gathered}
\text { Human Motor Control Section, National Institute of } \\
\text { Neurological Disorders and Stroke }
\end{gathered}
$$

Background: Essential tremor (ET) is a common movement disorder affecting $0.4 \%$ of the general population and up to 
$14 \%$ of people 65 years and older. Response to medications such as beta blockers and primidone may be of benefit, but are often accompanied by intolerable side effects. Response to ethanol, on the other hand, has a roughly $80 \%$ chance of significant tremor reduction, though daily use of this as a treatment has potentially serious medical, social, and legal consequences. Both ethanol and 1-octanol have been shown to reduce harmaline-induced tremor in rodents, an animal model of ET; however, 1-octanol does this at a dose much lower than that leading to intoxication, suggesting it may be useful in the treatment of essential tremor.

Methods: Our study population consists of adults with ethanol-responsive ET. Two formulations of 1-octanol have been utilized to date, a capsule containing 1-octanol adsorbed cellulose particles and a gelcap filled with 1-octanol in an oil-based vehicle. Primary outcome measures have included several measures including spiral drawings.

Results: Our initial findings have shown 1-octanol to be safe and effective in a placebo-controlled trial and in an open label dose-finding trial at doses up to $64 \mathrm{mg} / \mathrm{kg}$. Two initial formulations of 1-octanol have shown an unstable shelf-life and/or poor systemic absorption. We have failed so far to develop an assay for octanol that will permit pharmacokinetic studies.

Conclusions: Octanol is a promising drug for ET, but there are problems in its development. We need to develop a satisfactory assay and a good formulation in order to proceed with further clinical trials. Lacking some of the necessary skills, our $\mathrm{NIH}$ team has now partnered with industry to speed up progress.

\section{Identification of a Rapid-Throughput Battery of Pheontypic Tests for Drug Candidate Evaluation in the $\boldsymbol{\Delta} \mathbf{- 7}$ Mouse Model of Spinal Muscular Atrophy}

\author{
B El-Khodor, N Edgar, A Chen, MP Heyes \\ PsychoGenics Inc.
}

Spinal muscular atrophy (SMA) is characterized by selective loss of $\alpha$-motor neurons and is caused by homozygous loss or mutation in the telomeric survival motor neuron (SMN1) gene. Currently, there are no specific treatments for SMA. Drugs that improve $\alpha$-motor neuron survival and/or connectivity are potential therapeutic candidates. A high capacity and practical in vivo assessment system in the neonates (as early as the day of birth) is important to identify and evaluate therapeutic candidates. Key features of SMA are modeled in mice by deletion of the smn gene and insertion of the human SMN2 gene $\left(\mathrm{smn}^{-1-}\right.$; $\left.\mathrm{SMN} 2^{+/+}\right)$. Incorporation of the $\Delta 7$ gene into this SMA model improves survival into a more experimentally useful range of symptom progression, severity and drug administration protocols (see Le et al., Hum. Mol. Genet. 14: 845, 2005). In the present study, SMA model mice $\left(\mathrm{smn}^{-/-} ; \mathrm{SMN} 2^{+/+} ; \Delta 7^{+/+}\right.$) and control mice $\left(\mathrm{smn}^{+/+} ; \mathrm{SMN} 2^{+/+} ; \Delta 7^{+/+}\right)$were assessed daily for body weight and survival, and every other day for muscle tone, respiratory rate, gasping, degree of cyanosis, stomach milk content, and performance in a geotaxis test. Hind limb measures of strength, stamina, and general muscle tone were also evaluated. Around postnatal day 7, SMA mice showed lower body weights than controls and had a mean survival time of 13.5 days, as reported previously (Le et al., 2005). SMA mice did not acquire the ability to perform the geotaxis test and exhibited consistently hypotonic hind limbs. Other parameters were more variable and impaired at later time points. Furthermore, there was a significant correlation between body weight change and survival, which predicted the day of death within a 1-2 day period. This battery of tests in the $\Delta 7$ mouse model of SMA provides a rapid throughput and efficient means to identify, evaluate, and develop candidate therapies as a prelude to human clinical trials.

Supported by the Spinal Muscular Atrophy Foundation.

Reference: Le et al, Hum. Mol. Genet. 14: 845, (2005).

\section{Assessing the Signal Detection Properties of 3 Outcome Scales Used in Clinical Trials of Patients with Generalized Anxiety Disorder}

\section{Q Jiang, S Ahmed, R Pedersen, J Musgnung, R Entsuah Wyeth Pharmaceuticals}

Introduction: This analysis assessed correlations between the signal detection properties (drug vs placebo) of 3 outcome scales - the 14-item Hamilton Rating Scale for Anxiety (HAMA), the Clinician Global Impression of Severity (CGI-S), and the Clinical Global Impression of Improvement (CGI-I) - in generalized anxiety disorder (GAD) clinical trials.

Methods: Data from 5 randomized, double-blind, placebocontrolled venlafaxine XR studies in adult patients with GAD were pooled and examined individually. For all rating scales, Pearson correlation coefficients were calculated for all patients at each visit and by treatment arm. To evaluate signal detection properties, effect sizes and $P$ values based on the pooled and individual study data were examined for the 3 scales.

Results: At pretreatment visits, for the HAM-A and CGI-S, respectively, 1837 and 1831 observations were available, with mean scores of 25.8 and 4.5 , and the correlation coefficient between the 2 scales was $0.55(P<0.0001)$. Correlation coefficients at week 1 were 0.69 (HAM-A/CGI-S), 0.66 (HAMA/CGI-I), and 0.55 (CGI-S/CGI-I). Correlation coefficients increased each week, and at final visit were 0.83 (HAM-A/CGIS), 0.84 (HAM-A/CGI-I), and 0.82 (CGI-I/CGI-S). All correlations were significant $(P<0.0001)$ and were of comparable magnitude in the venlafaxine XR and placebo groups. Pooled effect sizes (venlafaxine XR versus placebo) were 0.37 , 0.41 , and 0.40 for HAM-A, CGI-S, and CGI-I, respectively (week $8 \mathrm{LOCF}$ ). Across studies, effect sizes ranged from 0.21 to $0.55,0.23$ to 0.68 , and 0.26 to 0.59 for the HAM-A, CGI-S, and CGI-I, respectively. As with the pooled data, however, within studies, they were more consistent across the 3 outcome measures. All 3 outcome measures reached statistical significance $(P<0.05)$ in 4 of 5 studies.

Conclusions: The 3 scales were consistently correlated in all studies, and the correlations increased during the conduct of the study. Effect sizes based on different scales in the same studies were more similar than effect sizes based on the same scale in different studies. Furthermore, no one scale stood out as having consistently better signal detection properties than the others.

Supported by Wyeth Pharmaceuticals.

\section{An Analysis of Correlations Among 4 Outcome Scales Employed in Clinical Trials of Patients With Major Depressive Disorder}

\section{Q Jiang, S Ahmed, R Pedersen, J Musgnung, R Entsuah} Wyeth Pharmaceuticals

Introduction: The objective of this analysis was to identify and assess any correlations among 4 widely used rating scales - the 17-item Hamilton Depression Rating Scale (HAM$\mathrm{D}_{17}$ ), the Montgomery-Asberg Depression Scale (MADRS), the Clinical Global Impression-Severity (CGI-S) and -Improve- 
ment (CGI-I) - in clinical trials with patients with major depressive disorder (MDD).

Methods: Data from 22 randomized, double-blind, placebocontrolled venlafaxine studies (10 ER studies, 11 IR studies, 1 with both formulations) in adult patients with MDD were pooled and examined from baseline through the first 8 weeks of treatment. For all rating scales, Pearson correlation coefficients were calculated between change scores and by treatment arm for patients at each visit. Correlations between binary outcomes (response defined as CGI-I and CGI-S $\leq 2,50 \%$ decrease in HAM-D ${ }_{17}$ and MADRS) were determined.

Results: At pretreatment visits, for the HAM-D ${ }_{17}$, MADRS, and CGI-S, respectively, 5117, 4871, and 5103 observations were available, with mean scores of 23.0, 29.1, and 4.4. Pretreatment correlations ranged from 0.52 (CGI-S and HAM$\left.\mathrm{D}_{17}\right), 0.53$ (CGI-S and MADRS), and $0.62\left(\mathrm{HAM}-\mathrm{D}_{17}\right.$ and MADRS). Correlations between scales increased at each visit and, at 8 weeks, ranged from 0.87 (CGI-S and CGI-I) to 0.93 (HAM-D ${ }_{17}$ and MADRS). Correlation coefficients in treatment arm subgroup analyses and between change scores were comparable. Correlation coefficients between binary outcomes were lower, ranging from 0.42 (CGI-I and CGI-S) to 0.61 (HAM$\mathrm{D}_{17}$ and MADRS) at week 1 and from 0.61 (CGI-I and CGI-S) to 0.81 (HAM-D ${ }_{17}$ and MADRS) at week 8. All correlation coefficients were significant $(P<0.0001)$.

Conclusions: Correlations among the four commonly used outcome scales were high; however, correlations among binary outcomes based on the scales were lower. The highest correlations were between the HAM-D ${ }_{17}$ and the MADRS, which share several items and have similar modes of administration and rating. The modest but consistently lower correlations between the CGI-S and CGI-I scales were unexpected because these scales are sometimes considered interchangeable.

Supported by Wyeth Pharmaceuticals.

\section{Imaging of Neurotransmitter Deficits: New Potential Biomarkers for Alzheimer's Disease?}

K Herholz, A Nordberg, J Masdeu, A Gerhard, K Ebmeier, S Pappata, D Perani, K van Laere, C Halldin, E Salmon, G Knudsen

Task Group on Early Diagnosis of Neurodegenerative Disease within the European Network of Excellence on Diagnostic Molecular Imaging (DiMI)

There is growing agreement that early diagnosis of Alzheimer's disease (AD) prior to onset of dementia is required for efficient clinical trials of neuroprotective interventions. In the past years, substantial progress has been made in that respect by refining clinical and neuropsychological assessment and quantitative evaluation of structural and functional brain imaging (mainly MRI and PET). It is also hoped that quantitative imaging will provide more efficient means to monitor disease progression than repeated neuropsychological testing. Alzheimer's disease is likely to be a common clinical manifestation with diverse and multifactorial etiology, and it also needs to be distinguished from other dementias, such as fronto-temporal dementia (FTD), dementia with Lewy bodies (DLB), and vascular dementia (VD). Yet, currently available clinical tools do not yet allow identifying specific etiologies and distinguishing between different dementing diseases at the clinical stage of mild cognitive impairment.

To address these needs, we have formed a multicentre network to compare molecular and neurotransmitter imaging in mild cognitive impairment. Within this network we are includ- ing normal controls and patients with mild cognitive impairment using harmonized longitudinal study protocols that share common neuropsychological and clinical assessment (MMSE, DemTect, CDR, Rey auditory verbal learning and complex figure, digit span, verbal fluency, trail making, IADL, Geriatric depression scale, NPI). Participating laboratories have established positron emission tomography (PET) or single photon emission computed tomography (SPECT) procedures to assess in vivo key molecular events, specifically amyloid deposition (C-11-PIB) and microglial activiation (C-11-PK11195), and assessment of neurotransmitter deficits, specifically acetylcholine esterase (C-11-MP4A, C-11-MP4P), nicotinic receptors (I-123-A85380), serotonin and benzodiazepine receptors, and dopamine transporters.

Preliminary results and evidence from postmortem studies suggest that amyloid deposition and cholinergic deficits occur early in $\mathrm{AD}$ but not in FTD, serotonergic deficits may be present in $\mathrm{AD}$ and FTD, whereas a dopaminergic deficit is seen in DLB only. The ongoing studies in MCI patients should provide more information on whether early differential diagnosis is possible and could become available for testing of drugs that target specific molecular mechanisms and transmitter systems.

Supported by the European Commission.

\section{Characterization of Soluble Fas Receptor as a Neuroprotective Agent following Acute Spinal Cord Injury \\ S Robins, M Fehlings \\ University of Toronto and University Health Network}

Spinal cord injury (SCI) is a devastating form of neurotrauma, affecting any population, and is a major cause of morbidity and mortality in children and young adults. The pathophysiology of SCI consists of a primary mechanical insult that triggers a secondary cascade of cellular damage. A key event in this pathology is delayed apoptotic cell death at and adjacent to the injury site, leading to progressive neurodegeneration. Several molecular pathways have been attributed to apoptosis after SCI. The Fas receptor pathway plays an integral role in the initiation of apoptosis through receptor-ligand binding of target cells and has been observed after CNS trauma and in several neurodegenerative diseases. Previous work in our lab has revealed that subarachnoid infusion of a soluble form of the Fas receptor (sFasR) is neuroprotective, as shown by long-term behavioral evaluation and neuronal tracing. We hypothesize that inhibition of the Fas receptor pathway is neuroprotective in the acutely injured spinal cord and results in reduction of neuronal and oligodendroglial cell death and enhanced axonal integrity across the lesion site. In this study, we used a $35 \mathrm{~g}$ and $50 \mathrm{~g}$ clip compression injury model at C7-T1 followed by intrathecal administration of sFasR using osmotic minipumps and catheterization at the site of injury. Using Western blotting and immunohistochemistry at 5 and 7 days following injury, our results reveal enhanced axonal preservation, enhanced survival of oligodendrocytes, and reduction in apoptotic cell death in sFasR-treated animals compared with controls. These results indicate that disruption of the Fas pathway in acute injury can lead to enhanced axonal and tissue preservation, which mirrors well with the improved long-term recovery observed previously in our lab. Moreover, this work shows the potential of soluble Fas receptor administration to be a therapeutic option for individuals suffering from acute SCI. 


\section{Evaluation of Polyethylene Glycol as a Neuroprotective Strategy for Acute Spinal Cord Injury \\ D Baptiste, M Fehlings \\ Toronto Western Research Institute and Krembil Neuroscience Centre}

The pathophysiology of traumatic spinal cord injury (SCI) involves abnormal activation of the proteolytical enzymes calpain- 1 and caspase- 3 . In the present study we examined the effect of a single intravenous injection of the putative neuroprotective compound polyethylene glycol (PEG) on cytoskeletal protection following cervical SCI in mature Wistar rats.

PEG (2000 Da; 30\% w/w in sterile lactate ringers (SLR)) or SLR vehicle was administered in the rat tail vein at times 1 or 4 hours after a $35 \mathrm{~g}$ clip extradural compression injury at C7/T1. To assess the neuroprotective efficacy of PEG in the acute setting postinjury, we measured the levels of dephosphorylated neurofilament 200 (NF200) together with the accumulation of spectrin break down product 150 (SBDP 150) at times 2, 4, 8, or 24 hours post-SCI using Western blot approaches to assess calpain-1 activity. Western blots were also used to assess caspase- 3 activity at 24 hours post-SCI via measurement of the accumulation of processed $17-\mathrm{kDa}$ caspase- 3 fragments. The mean \pm SEM relative optical density values for dephosphorylated NF200 corresponding to the SLR and PEG treatments at times $2,4,8$, and 24 hours post-SCI were $0.64 \pm 0.049$ and $0.48 \pm 0.166,0.3 \pm 0.028$ and $0.48 \pm 0.134,0.33 \pm 0.077$ and $0.46 \pm 0.076$, and $0.24 \pm 0.018$ and $0.38 \pm 0.040$, respectively. Moreover, the levels of dephosphorylated NF200 were significantly greater following PEG-treatment by 24 hours post-SCI $(p=0.0362)$. Moreover, PEG-mediated preservation of dephosphorylated NF200 was correlated with reduced calpain- 1 activity at 2 and 4 hours post-SCI. Furthermore, PEG treatment resulted in reduced levels of accumulated 17-kDa caspase- 3 fragments.

In conclusion, these data obtained in a clinically relevant model of cervical SCI suggest that PEG protects the injured cord by preserving cytoskeletal protein NF200, possibly through reducing calpain- 1 and caspase- 3 activities. Moreover, the protective effects afforded by PEG may occur within a relevant therapeutic window of opportunity, as the efficacy of PEG treatments occurred even when the drug was administered 4 hours postinjury. These data are critical in establishing the therapeutic potential for translation of PEG into the clinical arena.

\section{CT Scan Hematoma Volume: A Surrogate Endpoint for the Effect of Treatment in Intracerebral Hemorrhage}

\author{
BE Skolnick, ${ }^{*}$ SM Davis, ${ }^{\dagger}$ NC Brun, ${ }^{\ddagger}$ SE Mathew, ${ }^{*}$ \\ SA Mayer ${ }^{\S}$ \\ *Novo Nordisk Inc, Princeton, New Jersey; ${ }^{\dagger}$ Royal \\ Melbourne Hospital, Parkville, Australia; ${ }^{\ddagger}$ Novo Nordisk \\ A/S, Bagsvaerd, Denmark; ${ }^{\S}$ Columbia University, New York,
} New York, USA

Intracerebral hemorrhage ( $\mathrm{ICH})$ represents approximately $15 \%$ of all strokes, with no approved therapeutic interventions available. The 30 -day mortality rate is 35 to $50 \%$ and only 10 to $20 \%$ of survivors regain functional independence. Previous studies have indicated that substantial increase in hematoma volume occurs within the first hours after ICH onset. These studies also indicated that hematoma volume expansion is as- sociated with neurological deterioration and was a critical determinant of 30-day mortality. Early interventions directed at reducing hematoma expansion would be of considerable value. However, no validated measurements exist to directly translate the impact of hematoma volume on clinical outcomes. Changes in hematoma volume as determined by computed tomography (CT) scan is a potential surrogate endpoint for clinical outcomes. A recent randomized, double-blind, parallel-group, placebo-controlled clinical trial (399 patients) evaluated the use of rFVIIa (recombinant activated coagulation Factor VII) to reduce expansion of ICH hematoma volume, also determined the inter- and intrareader variability of CT scan measurements. In addition, assessments of clinical outcomes (modified Rankin Score, mRS) and mortality were conducted.

A baseline CT scan was performed within 3 hours after symptom onset. Placebo or rFVIIa was administered within 1 hour after the baseline scan. CT scans were repeated at 24 hours and 72 hours post-treatment. Inter-reader and intrareader variability was assessed by comparison of ICH volumes measured on CT images by two independent neuroradiologists, masked to treatment.

A meta-analysis conducted on a hemostatic treatment-naïve ICH patient population pooled from four studies $(N=218) \mathrm{dem}-$ onstrated that hematoma volume was an independent determinant of mortality $(p<0.0001)$ and $\mathrm{mRS}(p=0.0003)$. The results of a clinical trial using rFVIIa indicated that CT scan measurements of ICH volumes showed excellent intraclass correlations for interreader (0.9569) and intrareader variability (0.9844). Additionally, treatment with $\mathrm{rFVII}$ a resulted in significant reduction in hematoma volume $(p=0.01)$, mortality $(p=0.02)$ as well as improved clinical outcome $(p=0.004, \mathrm{mRS})$ for rFVIIa-treated patients compared to placebo. Therefore, change in hematoma volume as determined by CT scan has potential as a clinically relevant surrogate measure, with value in the study of early hemostatic interventions in acute neurologic settings where brain hemorrhage is of significant concern.

\section{Androstenol (5 $\alpha$-androst-16-en-3 $\alpha$-ol) Is a Novel Neurosteroid Positive Modulator of GABA $_{\mathrm{A}}$ Receptors: In Vitro and In Vivo Studies}

\author{
RM Kaminski, ${ }^{*}$ H Marini, ${ }^{\dagger}$ PI, Ortinski, ${ }^{\ddagger}$ W Yonekawa,* \\ S Vicini, ${ }^{\ddagger}$ MA Rogawski* \\ *Epilepsy Research Section, National Institute of \\ Neurological Disorders and Stroke, National Institutes of \\ Health, Bethesda, Maryland; ${ }^{\dagger}$ Faculty of Medicine and \\ Surgery, A.O.U.G. Martino, University of Messina, Messina, \\ Italy; ${ }^{\ddagger}$ Department of Physiology and Biophysics, \\ Georgetown University School of Medicine
}

Androstenol ( $5 \alpha$-androst-16-en- $3 \alpha$-ol) is a volatile steroid compound belonging to the group of 16-androstenes found in human plasma. It is structurally similar to endogenous A-ring reduced steroids that act as positive modulators of $\mathrm{GABA}_{\mathrm{A}}$ receptors, i.e., neurosteroids. Thus, we have hypothesized that androstenol may have electrophysiological and behavioral traits characteristic for neurosteroids.

The influence of androstenol on $\mathrm{GABA}_{\mathrm{A}}$ receptors currents under voltage clamp conditions in mouse cerebellar granule cell cultures, rat brain slices, and HEK cells expressing human $\mathrm{GABA}_{\mathrm{A}}$ receptor subunits has been assessed. Additionally, the effect of androstenol on 4-aminopyridine-induced epileptiform activity in rat hippocampal slices was studied. Assessment of anticonvulsant, anxiolytic, and antidepressant effects of androste- 
nol in the $6 \mathrm{~Hz}$, PTZ, open field, and forced swim models has also been performed.

We have found that androstenol potentiates GABA-evoked currents in HEK cells as well as in cerebellar cultures and slices. Androstenol inhibited epileptiform activity induced by 4-aminopyridine in rat brain slices and had strong anticonvulsant effects against PTZ- and $6 \mathrm{~Hz}$-induced seizures in mice. In addition, we have found that androstenol has anxiolytic and antidepressant effects in animal models.

These results for the first time demonstrate that androstenol acts as a positive modulator of $\mathrm{GABA}_{\mathrm{A}}$ receptors and has behavioral properties compatible with this physiological action. Androstenol may act as an endogenous modulator of $\mathrm{GABA}_{\mathrm{A}}$ receptors, and it may be useful in treatment of epilepsy and possibly other neurological disorders, i.e., anxiety and depression.

Supported by NINDS/NIH.

\section{Prolonged Attenuation of Amygdla Kindled Seizures by Local Delivery of Large Molecular Weight Therapeutic Agents by Convection- Enhanced Delivery in Rats}

\author{
M Gasior, R Tang, N White, MA Rogawski \\ Epilepsy Research Section, NINDS, NIH
}

Many patients with temporal lobe epilepsy (TLE) respond inadequately to antiepileptic drugs, necessitating resective surgery as a last resort. An alternative less invasive approach is site-specific delivery of therapeutic agents into the epileptic focus. However, conventional intraparenchymal injection produces localized tissue damage and there is poor control over the drug distribution. Convection-enhanced delivery (CED) is an alternative infusion technique that allows for the nondestructive and well-controlled localized delivery of solutes, including high molecular weight agents (e.g., peptides, proteins, and even viral vectors). The present study examined whether long-term changes in amygdala-kindled seizure susceptibility can be produced by a one-time CED infusion into the amygdala. Agents selected for study were presynaptic neurotoxins capable of producing long-term attenuation of neurotransmitter release ei- ther by inhibiting $\mathrm{N}$-type calcium channels (i.e., $\omega$-conotoxins GVIA and MVIIA) or cleaving intracellular proteins involved in exocytosis (i.e., botulinum toxins type A and B). A conventional, small-molecule antiepileptic drug, carbamazepine, was used for comparison.

Each rat was implanted with a combination infusion cannula and stimulation electrode into the right basolateral amygdala. Daily stimulations were carried out until the animals were fully kindled (stage 5 seizures for at least five consecutive days). Then, the rats received infusions of vehicle alone or $\omega$-conotoxins $(5-500 \mathrm{pmol})$, botulinum toxins (7-67 fmol), or carbamazepine (500 nmol) through the cannula whose tip was adjacent to the stimulation site. Each dose was delivered in a volume of $5 \mu \mathrm{L}$ at a rate of 0.25 $\mu \mathrm{L} / \mathrm{min}$. Electrophysiological (afterdischarge threshold and duration) and behavioral (seizure stage and duration) measures of amygdala-kindled seizures were recorded daily for up to 64 days after the infusion.

There were no changes in any of the kindling parameters in rats that had received vehicle infusions. In contrast, infusions of $\omega$-conotoxins and botulinum toxins resulted in a dose- and time-dependent increase in the afterdischarge threshold and a decrease in seizure stage and duration, indicting an inhibitory effect on kindled seizure expression. The protective effects of $\omega$-conotoxins reached a maximum at $48 \mathrm{~h}$ postinfusion and then gradually dissipated within the next five days; in contrast, effects of botulinum toxins lasted for several weeks. Infusions of each toxin appeared well tolerated and devoid of any immediate or long-term side effects. In marked contrast to the longlasting effects produced by $\omega$-conotoxins and botulinum toxins, attenuation of seizures produced by carbamazepine lasted only a few hours after the infusion.

These results indicate that local CED-mediated delivery of high molecular weight presynaptic toxins can produce longlasting anticonvulsant effects that persist for weeks. Whether this approach will be useful in the treatment of human epilepsy or other brain disorders requires further investigation.

Supported by NINDS, no conflict of interest.

\section{Invited Posters: Participating Member Organizations of the Advocacy Forum}

\section{Dystonia Medical Research Foundation: 30 Years of Promoting Research and Therapy Development}

Dystonia is a neurological disorder of movement. It is characterized by involuntary contractions of various parts of the body causing discomfort and pain and, in more severe forms, incapacitating the affected individuals. Although the exact cause of the disease in unknown, several forms known as primary dystonias are inherited. Other, secondary dystonias result from brain injuries or exposure to toxic drugs. Dystonia often accompanies other neurological diseases. In North America alone, more than 300,000 people are affected by this disease, making it the third most common movement disorder. Due to its nature, the psychological and social impact of the dystonias is enormous. It is estimated that the total annual cost to the national economy can be counted in billions of dollars.

Thirty years ago, in response to the growing and unfulfilled needs of the dystonia community, the Dystonia Medical Research Foundation (DMRF) was established. One of the major goals of the Foundation is to promote and support basic and clinical research into the causes and mechanisms of the dystonias. The Foundation also supports efforts which aim at discovery and development of novel treatments and therapies. Since its founding, the DMRF-funded researchers have made major discoveries in the field. These efforts culminated in 1997, when the first dystonia-causing gene, DYT1, was cloned. Genetic studies indicate that there are at least 14 other genes causing inherited forms of the disease. The implications of gene discoveries are obvious. TorsinA, the product of DYT1 gene, has been extensively characterized. The knowledge about its structure, cellular localization, interactions, and physiological function provides an opportunity to identify viable targets for drug development. To explore these exciting possibilities, the DMRF began funding research aiming at the development of novel therapeutic agents and established the Cure Dystonia Initiative (CDI). The major goal of the CDI is to accelerate the development of more effective treatments for dystonia with the ultimate goal of finding a cure. The CDI is envisioned as a bridge between basic and translational research. As the CDI is still at its early stages, several research groups supported by the 
DMRF have already begun testing promising drug candidates which target pathogenic genes and proteins. Faithful to its tradition, the DMRF is committed to support basic and clinical research, as well as therapeutics development.

\section{Survey on Patient and Physician Attitudes about Parkinson Clinical Trials}

In March 2005, Harris Interactive ${ }^{\circledR}$ completed a study examining physicians' and patients' attitudes towards Parkinson's disease clinical trials. The study was commissioned on behalf of PDtrials* by the Michael J. Fox Foundation for Parkinson's Research.

Five hundred physicians (250 primary care physicians/gerontologists and 250 neurologists) and 518 people with Parkinson's participated in the study. Nearly $80 \%$ of patients surveyed stated that they would be somewhat, very, or extremely likely to participate in a clinical trial if one were available in their area. However, seven in ten are not at all or not very aware of clinical trials and only $11 \%$ reported that their doctor ever suggested that they participate in a trial.

The survey also found that, among physicians who treat people with Parkinson's, 96\% agree that clinical trials are necessary to find better treatments for the disease. However, the majority have discussed clinical trials with just $10 \%$ or less of their patients and never referred a patient to a clinical trial.

Only $14 \%$ of primary care physicians, $21 \%$ of neurologists, and $18 \%$ of patients surveyed indicated that they are somewhat or very satisfied with the amount of information available about clinical trials for Parkinson's disease.

The Harris Interactive study confirms the assumption that there is a clinical trials information gap and the critical role of PDtrials, a national initiative dedicated to increasing education and awareness about clinical research by providing information to help people with Parkinson's learn and make informed decisions about participating in clinical trials.

*PDtrials is led by the Parkinson's Disease Foundation in collaboration with the American Parkinson Disease Association, The Michael J. Fox Foundation for Parkinson's Research, the National Parkinson Foundation, the Parkinson's Action Network, The Parkinson Alliance and WE MOVE. The campaign is advised by the National Institute of Neurological Disorders and Stroke (NIH), the Parkinson Study Group, the Parkinson Pipeline Project and the Parkinson's Institute.

\section{www.PDtrials.org: Increasing Parkinson's Clinical Trial Awareness through the Internet}

PDtrials* is a national initiative dedicated to increasing education and awareness about clinical research by providing information to help people with Parkinson's learn and make informed decisions about participating in clinical trials. www.PDtrials.org is a first-of-its-kind website that lists over 50 clinical studies seeking participants. It sees over 25,000 visits per month and has an email list of close to 2,500.

PDtrials was initiated in response to a concern within the Parkinson's community about the low levels of awareness and participation in clinical research. As many as one million people in the U.S. live with Parkinson's yet less than one percent participate in clinical studies-far short of volunteers that researchers anticipate will be needed over the next two to three years.

In October 2005, the PDtrials campaign initiated a series of online marketing strategies to increase visibility for www. PDtrials.org. These included the development of a monthly HTML email newsletter-the PDtrials Bulletin and engaging in "viral" marketing, such as forwarding the PDtrials Bulletin to contacts throughout the community.

The results were an increase in average daily visitors from 465 in September 2005 to 822 in January 2006 and an increase in overall visits from 13,956 in September 2005 to 25,506 in January 2006. In addition, over $50 \%$ of PDtrials Bulletin recipients opened the email and clicked through from the Bulletin to www.PDtrials.org. This compares with a nonprofit industry standard of a $30 \%$ open rate and $3 \%$ click-through rate.

Various strategies have been employed to increase web traffic to www.PDtrials.org. The greatest success has been through online cultivation of individuals who have requested to receive information about clinical studies and who are thought to be interested.

*PDtrials is led by the Parkinson's Disease Foundation in collaboration with the American Parkinson Disease Association, The Michael J. Fox Foundation for Parkinson's Research, the National Parkinson Foundation, the Parkinson's Action Network, The Parkinson Alliance and WE MOVE. The campaign is advised by the National Institute of Neurological Disorders and Stroke (NIH), the Parkinson Study Group, the Parkinson Pipeline Project and the Parkinson's Institute. 